АКУUЕР СТВ
ГИЕКОЛОГИЯ
РЕПРОДУКЦИЯ Включен в перечень ведущих рецензируемых журналов и изданий ВАК

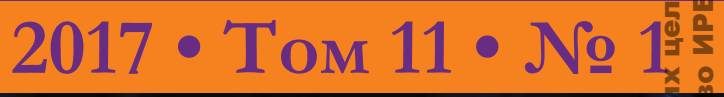

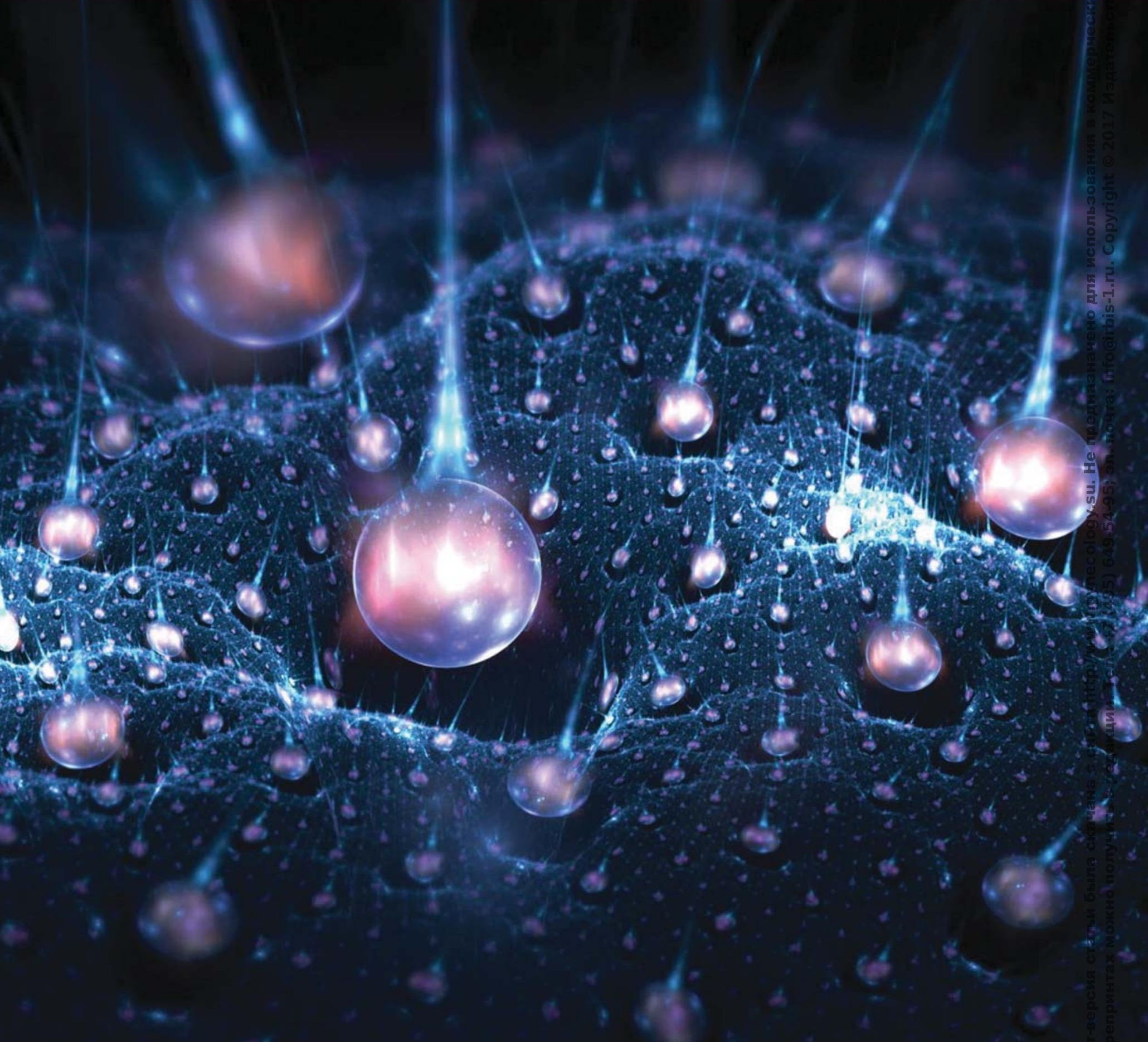

\title{
OBSTETRICS, GYNECOLOGY AND REPRODUCTION!
}




\title{
СРАВНИТЕЛЬНАЯ ХАРАКТЕРИСТИКА ДИФФУЗИОННО-ВЗВЕШЕННЫХ ПОСЛЕДОВАТЕЛЬНОСТЕЙ МРТ И МУЛЬТИСПИРАЛЬНОЙ КОМПЬЮТЕРНОЙ ТОМОГРАФИИ В СТАДИРОВАНИИ РАКА ЯИЧНИКОВ
}

\author{
Солопова А.Е., Терновой С.К., Алипов В.И., Макацария А.Д.
} ФГАОУ ВО Первый МГМУ им. И.М. Сеченова Минздрава России (Сеченовский Университет)

\begin{abstract}
Резюме
Цель - сравнить диагностические возможности комплексного МРТ-исследования с дисффузионно-взвешенными последовательностями (ДВ-МРТ) и мультиспиральной компьютерной томограсьии (МСКТ) в рамках стадирования злокачественных новообразований (3НО) яичников. Материалы и методы. Исследование проводилось с февраля по декабрь 2016 г. 73 пациентки с подозрением на ЗНО яичников, которым было выполнено предоперационное обследование, включавшее МСКТ и ДВ-МРТ брюшной полости и малого таза на 1.5 Тл томографре c b-фракторами 0,1000 с/мм² и построением карт измеряемого коэфффициента дифффузии (ИКД). По полученным данным был рассчитан индекс перитонеального канцероматоза (ИПК) и построены ROC-кривые для ДВ-МРТ и МСКТ. Для верифрикации были использованы результаты хирургического стадирования и гистопатологического исследования операционного материала. Результаты. Были определены следующие стадии 3HO: 5 (6,8\%) IV; 35 (47,9\%) IIIC; $23(31,5 \%)$ IIIB; 10 (13,6\%) IIIA. Общая чувствительность составила 55\% для МСКТ и 77\% для ДВ-МРТ. В частности, ДВ-МРТ значительно превосходила МСКТ при обнаружении имплантации в брыжейку толстого кишечника (83,8 и $37,8 \%$ соответственно), серозную оболочку тонкого кишечника $(77,8$ и 28,9\%) и парааортальные лимфратические узлы (ЛУ) (71,4 и 35,7\%). Специфичность методов лучевой диагностики оказалась схожей $(79,6 \%$ - ДВ-МРТ и 81\% - МСКТ). Индекс перитонеального канцероматоза (ИПК), рассчитанный по данным ДВ-МРТ, коррелировал с данными хирургического вмешательства сильнее, чем ИПК по данным МСКТ ( $r=0,706$ для ДВ-МРТ, $r=0,544$ для MCKT). На основании полученных данных были построены ROC-кривые для ДВ-MPT и MCKT (площадь под кривой - соответственно 0,913 и 0,655). Было обнаружено снижение ИКД во вторичных опухолевых очагах $(1,120 \pm 0,25$

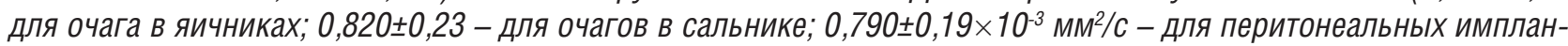
тов). Заключение. ДВ-МРТ значительно превосходит МСКТ по чувствительности и позволяет дать точную оценку распространенности опухолевого процесса.
\end{abstract}

\section{Ключевые слова}

MPT, дифрфуузионно-взвешенные изображения, злокачественные опухоли яичников, стадирование.

Статья поступила: 16.01.2017 г.; в доработанном виде: 27.02.2017 г.; принята к печати: 21.03.2017 г.

Конфликт интересов

Авторы заявляют об отсутствии необходимости раскрытия финансовой поддержки или конфликта интересов в отношении данной публикации.

Все авторы сделали эквивалентный вклад в подготовку публикации. 


\title{
Для цитирования
}

Солопова А.Е., Терновой С.К., Алипов В.И., Макацария А.Д. Сравнительная характеристика дифффузионно-взвешенных последовательностей МРТ и мультиспиральной компьютерной томографиии в стадировании рака яичников. Акушерство, гинекология и репродукция. 2017; 11 (1): 19-25. DOI: 10.17749/2313-7347.2017.11.1.019-025.

\section{OVARIAN CANCER STAGING: COMPARISON BETWEEN DIFFUSION-WEIGHTED MRI AND MULTISPIRAL COMPUTED TOMOGRAPHY}

\author{
Solopova A.E., Ternovoi S.K., Alipov V.I., Makatsariya A.D. \\ I.M. Sechenov First Moscow State Medical University, Ministry of Health of Russia, Moscow
}

\section{Summary}

The aim is to evaluate the diagnostic potential of the diffusion-weighted MRI sequences (DW-MRI) as compared with multi-spiral computed tomography (MSCT) for staging of ovarian malignancies (OVM). Materials and Methods. The study was conducted (from February to December 2016) on 73 patients with suspected ovarian malignancies who underwent preoperative examination, including abdominal / pelvic MSCT and DW-MRI, using a 1.5T scanner with $b$-factors of $0.1000 \mathrm{~s} / \mathrm{mm}^{2}$ and the calculation of apparent diffusion coefficient (ADC). Using the obtained data, the peritoneal cancer index (PCI) was calculated and ROC curves for DW-MRI vs MSCT were created. The imaging-based diagnoses were then compared with surgical staging and histopathological examination. Results. The following stages of OVM were determined: 5 (6.8\%) IV; 35 (47.9\%) IIIC; $23(31.5 \%)$ IIIB; $10(13.6 \%)$ IIIA. The overall sensitivity was $55 \%$ for MSCT and $77 \%$ for DW-MRI. For example, DW-MRI was markedly superior to MSCT in detecting a tumor implantation into the large intestine mesentery ( $83.8 \%$ and $37.8 \%$, respectively), into the small intestine serosa $(77.8 \%$ and $28.9 \%)$, and the para-aortic lymph nodes (71.4 and 35.7\%). The specificity of the compared imaging modalities was similar $(79.6 \%$ - DW-MRI and $81 \%$ - MSCT). The peritoneal cancer index (PCI) calculated from the DW-MRI results correlated with the surgical staging data better than the PCI based on MSCT ( $r=0.706$ for DW-MRI, $r=0.544$ for MSCT). Based on the obtained data, the ROC curves were plotted to show the areas under the curves of 0.913 and 0.655 for DW-MRI and MSCT, respectively. A decrease in the ADC values was found in secondary tumor foci $(1.120 \pm 0.25$ for the ovaries, $0.820 \pm 0.23$ for the omentum, $0.790 \pm 0.19 \times 10^{-3} \mathrm{~mm}^{2} / \mathrm{s}$ for peritoneal implants). Conclusion. For the assessment of ovarian cancer spread, DW-MRI is significantly more sensitive than MSCT.

\section{Key words}

MRI, diffusion-weighted images, malignant ovarian tumors, staging.

Received: 16.01.2017; in the revised form: 27.02.2017; accepted: 21.03.2017.

\section{Conflict of interests}

The authors declared that they do not have anything to disclosure regarding funding or conflict of interests with respect to this manuscript.

All authors contributed equally to this article.

\section{For citation}

Solopova A.E., Ternovoi S.K., Alipov V.I., Makatsariya A.D. Ovarian cancer staging: comparison between diffusion-weighted MRI and multispiral computed tomography. Akusherstvo, ginekologiya i reproduktsiya / Obstetrics, gynecology and reproduction. 2017; 11 (1): $19-25$ (in Russian). DOI: 10.17749/2313-7347.2017.11.1.019-025.

\section{Corresponding author}

Address: ul. Trubetskaya, 8-1, Moscow, Russia, 119991.

E-mail: dr.solopova@mail.ru (Solopova A.E.).

\section{Введение}

Злокачественные опухоли яичников (3НО) занимают 7-е место (5\%) в онкологической заболеваемости женского населения России, пятое - среди причин женской смертности от онкологии и первое - среди злокачественных образований органов малого таза [1]. Причиной высокой смертности пациенток с 3НО является поздняя диагностика примерно в 70\% случаев на стадиях III-IV, когда имеет место метастазирование опухоли по брюшине. При распространенном поражении уменьшается вероятность полного удаления опухоли, увеличивается риск рецидива и вероятность развития химиорезистентности [2]. Пятилетняя выживаемость пациенток с 3 НО варьирует от $92 \%$ на стадии I-II до 27\% на поздних стадиях [1]. 3НО включают в себя эпителиальные опухоли, собственно рак яичников (ЭРЯ), и неэпителиальные опухоли.

В настоящее время парадигмой является общность интраэпителиального рака фаллопиевых труб, ЭРЯ и перитонеального рака, и эти патологии рассматрива- 
ются как разные стадии одного процесса [3-6]. Для определения стадии используется классификация FIGO и TNM [7].

Лечение распространенного рака яичников проводят комбинацией хирургического вмешательства и химиотерапии. Последовательность применения этих методов зависит от стадии опухолевого процесса, возраста и общего состояния пациентки, сракторов, связанных с материально-техническим обеспечением клиники [8].

На стадиях опухоли II-IV цель хирургического вмешательства состоит в максимально возможном удалении опухолевой ткани. Согласно рекомендациям ESMO, полной циторедукцией считается выполнение экстирпации матки с яичниками и маточными трубами, удаление большого сальника и всех видимых проявлений опухолевого процесса без макроскопически определяемых остаточных опухолевых масс. Циторедукция считается оптимальной, если размер оставшихся опухолевых очагов не превышает 1 см и/или их количество превышает 20 [9].

Подобное деление связано с различным прогнозом дальнейшего течения заболевания. Проведение полной циторедукции приводит к увеличению медианы выживаемости в среднем на 2,5 мес., оптимальной - на 1,8 мес., а неоптимальной - не улучшает прогноз вовсе [10].

Таким образом, для точного стадирования и определения оптимальности проводимой циторедукции метод визуализации должен обнаруживать небольшие по размеру (5-10 мм) опухолевые импланты в брюшину, метастазы в паренхиматозные органы и в лимсратические узлы.

МСКТ достаточно хорошо обнаруживает пораженные лимсратические узлы и метастазы в паренхиму органов [11,12], однако ряд исследований показал их низкую чувствительность при обнаружении опухолевых очагов <1см (чувствительность МСКТ составляла 25-55\%) [13,14].

Ряд исследователей показал высокую чувствительность и специфичность ДВ-МРТ при обнаружении небольших перитонеальных имплантов ЗНО, а также высокую чувствительность, но низкую специфичность при оценке лимфратических узлов (предоперационная оценка распространения опухоли по брюшине с помощью ДВ-МРТ имела точность от 80 до 100\%) [17,18].

Ограничение дифффузии в тканях можно количественно оценить с помощью измеряемого коэффиициента дифффузии (ИКД). Чем ниже его значение, тем сильнее ограничена дифффузия жидкости в данной области. ИКД можно использовать для визуальной диагностики (карты ИКД), для диффреренциальной диагностики злокачественных и доброкачественных образований, а также для прогнозирования ответа опухоли на проводимую химиотерапию $[15,16]$.

Кроме того, активно исследуется возможность внедрения ДВ-МРТ всего тела с целью комплексной оценки состояния внутренних органов и обнаружения отдаленных метастазов 3 НО [17].

Целью исследования является сравнительная характеристика диагностических возможностей комплексного МРТ-исследования с ДВ-последовательностями при стадировании $3 \mathrm{HO}$ яичников относительно МСКТ.

\section{Материалы и методы}

Исследование проводилось с февраля по декабрь 2016 г. В ГБУЗ «ОКД №1 ДЗМ» Г. Москвы. В нем приняли участие 73 пациентки с подозрением на $3 \mathrm{HO}$, которым было проведено комплексное клинико-лучевое исследование в рамках предоперационной оценки опухолевого процесса.

В качестве критериев включения выступали: подозрение на злокачественное новообразование яичников по данным фризикального исследования и данным УзИ органов малого таза; высокий уровень СА-125 (>35 Ед/мл).

В качестве критериев исключения выступали: наличие аллергической реакции на контрастное вещество; почечная недостаточность (клиренс креатинина $<40$ мл/мин); противопоказания к проведению МРТ.

Было выполнено комплексное МРТ-исследование, включавшее ДВ-последовательности, и МСКТ брюшной полости и малого таза. Полученные результаты были верисрицированы при гистопатологическом исследовании операционного материала. Семь пациенток $(0,95 \%)$ не подвергалась хирургическому лечению в связи с невозможностью проведения оптимальной циторедукции (пациентки с IV стадией и с критериями исключения ESUR [12]). Верисикация диагноза у них проводилась путем динамического наблюдения не менее 6 мес.

Пациентки воздерживались от еды перед исследованием в течение 3 ч. Для уменьшения перистальтики использовались холинолитики. Больные были проинструктированы относительно правильной техники дыхания с целью снижения количества артефактов от движения передней брюшной стенки.

Исследование проводилось при напряжении магнитного поля 1,5 Тл, с использованием 32-канальной поверхностной фазированной катушки на аппарате Vantage Atlas (Toshiba Medical System).

Исследование выполняли в положении пациентки на спине, с прямыми ногами. На область малого таза накладывали широкий пояс шириной 25-30 см - для фриксации передней брюшной стенки. Перед исследованием проводили катетеризацию вены с последующей установкой катетера и подсоединением инжектора.

ДВ-МРТ-исследование производилось при значениях b-срактора 0 и 1000 c/mм² в поперечной плоскости. Общее время МРТ-исследования составляло в среднем 40 мин.

Для получения ДВИ использовали сверхбыстрые одноимпульсные (single shot) эхо-планарные последовательности без подавления сигнала от свободной 
жидкости с парой дополнительных дифффузионных градиентов (десразирующего и ресразирующего) одинаковой амплитуды и длительности.

Для повышения воспроизводимости (снижение влияния других параметров) в автоматическом режиме определяли измеряемый коэфрфициент дифффузии (ИКД) - количественный параметр дифффузии, не зависящий от времен спин-спиновой и спин-решетчатой релаксации.

Отдельно для каждого метода лучевой диагностики оценивалась степень вовлеченности брюшины в патологический процесс и рассчитывался индекс перитонеального канцероматоза (ИПК) [18]. При этом рассматривались 13 областей брюшной полости и размер опухолевых очагов (от 0 до 3 баллов, в зависимости от размера очага). ИПК может принимать значения от 0 до 39, рассчитываясь по формуле:

$$
\text { ИПК }=P O \times N \text {, }
$$

где РО - размер опухолевых очагов; N - число анатомических областей.

ИПК позволяет количественно оценить степень распространенности процесса по брюшине. Значения от 0 до 20 говорят об умеренном распространении, а от 20 и выше - о значительной вовлеченности брюшины в опухолевый процесс [18].

\section{Результаты}

Были определены следующие стадии 3НО: 5 (6,8\%) IV; 35 (47,9\%) IIIC; 23 (31,5\%) IIIB; 10 (13,6\%) IIIA.

После интерпретации данных лучевой диагностики и верификации диагноза была произведена оценка чувствительности (Ч) и специфичнности (С) КТ и ДВ-МРТ при обнаружении опухолевых метастазов в различные области. Полученные результаты представлены в таблице 1.

Таким образом, ДВ-МРТ обладает значительно большей чувствительностью, чем МСКТ при обнаружении опухолевых имплантационных метастазов по брюшине и брыжейке (по современной анатомической классисрикации считается отдельным органом брюшной полости [19]) кишечника, при этом специфиччность метода была незначительно ниже.

Средние ИПК, по данным хирургического вмеша-

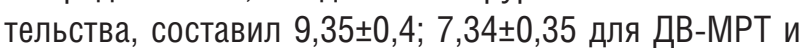
$3,75 \pm 0,4$ для МСКТ. Был проведен статистический тест Пирсона, чтобы оценить корреляции значений ИПК для методов лучевой диагностики и данных хирургического вмешательства. В результате для ДВ-МРТ $r=0,706$ (высокая корреляция; $P<0,001$ ), для МСКТ $r=0,544$ (значимая корреляция; $P<0,001$ ).

\begin{tabular}{|c|c|c|c|c|c|c|c|c|}
\hline \multirow{2}{*}{ Метод визуализации } & \multicolumn{4}{|c|}{ MCKT } & \multicolumn{4}{|c|}{ ДВ-МРТ } \\
\hline & 4 & C & ПП3 & $0 \Pi 3$ & 4 & C & ПП3 & $0 \Pi 3$ \\
\hline $\begin{array}{l}\text { Серозная оболочка } \\
\text { тонкого кишечника }\end{array}$ & 28,9 & 78,6 & 68,4 & 40,7 & 77,8 & 75 & 83,4 & 67,7 \\
\hline Брыжейка тонкого кишечника & 41,9 & 86,7 & 81,8 & 51 & 79,1 & 73,3 & 81 & 71 \\
\hline $\begin{array}{l}\text { Серозная оболочка } \\
\text { толстой кишки }\end{array}$ & 50 & 81,8 & 72 & 62,5 & 86,1 & 73 & 24,4 & 84,4 \\
\hline Брыжейка толстой кишки & 37,8 & 83,3 & 70 & 56,6 & 83,8 & 72,2 & 75,6 & 81,3 \\
\hline Сальник & 86,7 & 53,6 & 75 & 71,4 & 75,6 & 75 & 82,9 & 65,6 \\
\hline Желудочно-печеночная связка & 75 & 78,8 & 81,8 & 72,2 & 55 & 87,9 & 84,6 & 61,7 \\
\hline Сумка Моррисона & 64,1 & 85,3 & 67,4 & 83,3 & 87,2 & 76,5 & 83,9 & 81 \\
\hline Селезенка & 51,6 & 83,3 & 69,6 & 70 & 58,1 & 88,1 & 78,3 & 74 \\
\hline Диафрагма слева & 59,5 & 77,8 & 73,3 & 65,1 & 89,2 & 90 & 89,1 & 88,9 \\
\hline Диафрагма справа & 47,7 & 93,1 & 91,3 & 54 & 84,1 & 96,1 & 97,4 & 80 \\
\hline Брюшина мочевого пузыря & 89,1 & 78 & 92,5 & 70 & 90,9 & 83 & 94,3 & 75 \\
\hline Поверхность печени & 57,6 & 80 & 70,4 & 69,6 & 72,7 & 65 & 63,2 & 74,3 \\
\hline Паренхима печени & 50 & 94,2 & 33,3 & 97 & 50 & 95,7 & 40 & 97,1 \\
\hline лУ малого таза & 50 & 67,9 & 37 & 78,3 & 90 & 69,8 & 52,9 & 94,9 \\
\hline Парааортальные ЛУ & 35,7 & 88,1 & 41,7 & 85,2 & 71,4 & 72,9 & 38,5 & 91,5 \\
\hline
\end{tabular}

Таблица 1. Показатели информативности методов лучевой диагностики.

Примечание. Ч - чувствительность, С-специфичность, ППЗ - положительное прогностическое значение, ОПЗ - отрицательное прогностическое значение.

Table 1. Parameters of diagnostic imaging.

Note. 4 - sensitivity, C-specificity, ПП3-positive prognostic value, ОП3-negative prognostic value. 
Единые критерии возможности проведения оптимальной циторедукции отсутствуют. Однако ИПК>20 свидетельствует о невозможности проведения оптимальной циторедукции, а также ассоциирован с высоким риском интраоперационных осложнений и послеоперационной летальности [20,21]. На основании этого критерия была произведена оценка способности ДВ-МРТ и МСКТ определять возможность проведения оптимальной циторедукции. Для методов были подсчитаны площади под кривой (area under the curve, AUC). Для ДВ-МРТ значение составило 0,913, а для МСКТ - 0,655. В качестве контроля выступал ИПК, установленный по данным хирургического вмешательства.

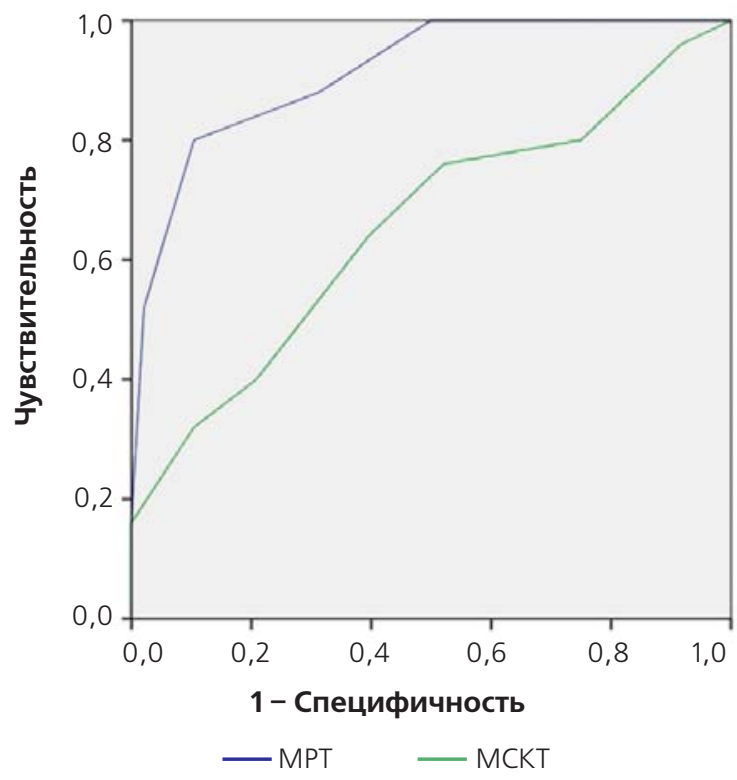

Рисунок 1. ROC-кривые для МРT с диффузионновзвешенными последовательностями (МРТ) и компьютерной томографии (МСКТ).

Оиенивали способность определять возможность оптимальной ицторедукиии. Площадь под кривой $M P T=0,913, M C K T=0,655$.

Figure 1. ROC curves for MRI with diffusion-weighted sequences (MRI) and for computed tomography (MSCT).

Comparing these two imaging modalities, indications for optimal cytoreduction were assessed. The areas under the curves: $M P T=0.913, M C K T=0.655$.

Кроме того, была проведена оценка ИКД в перитонеальных метастазах и в первичных опухолевых очагах. Среднее значение ИКД в перитонеальных имплантационных метастазах $\left(0,820 \pm 0,23 ; 0,790 \pm 0,19 \times 10^{-3} \mathrm{~mm}^{2} / \mathrm{c}-\right.$ большой сальник и брюшина, соответственно) было достоверно ниже $(\mathrm{P}<0,001)$, чем в первичной опухоли $\left(1,120 \pm 0,25 \times 10^{-3} \mathrm{Mm}^{2} / \mathrm{c}\right)$. При этом, несмотря на то что среднее значение ИКД для сальниковых метастазов было выше, чем для брюшинных, достоверного различия между ними не выявлено.

\section{Обсуждение}

Для оптимального и эфрфективного хирургического вмешательства и определения тактики ведения пациентки с ЭРЯ необходимо максимально точно оценить вовлеченность в опухолевый процесс брюшины, брыжейки, паренхиматозных органов и ЛУ.

Ряд исследований показали большую точность МРТ по сравнению с МСКТ в стадировании ЗНО. Так, в исследовании Low R.N. et al. проводилась оценка вовлеченности брюшины в опухолевый процесс у 22 пациентов с карциномой аппендикса и яичников с помощью МРТ и МСКТ. Согласно результатам чувствительность и специфичность методов составила соответственно 95 и 70\% для МРТ и 55 и 86\% для МСКТ. При этом ИКБ, вычисленный с помощью МРТ, в 91\% случаев соответствовал степени канцероматоза, определенной во время операции, в то время как для МСКТ ИКБ совпал лишь в 50\% [22]. Michielsen et al. проводили сравнение ДВ-МРТ всего тела, МСКТ и ПЭТ/КТ - по способности обнаружить опухолевые имплантационные метастазы в брюшину и поражение ЛУ брюшной полости и малого таза. В результате наибольшая чувствительность $(71,3 \%)$ была у ДВ-МРТ, тогда как у ПЭТ/КТ - 70\% и лишь 55,8\% - у MCKТ. При этом имплантацию в брыжейку толстой и тонкой кишки и опухолевые очаги в брюшной полости меньше 1 см с помощью МСКТ обнаружить не удалось вовсе, с помощью ДВ-МРТ очаги в брыжейку выявлялись с чувствительностью около 75\% [23]. Результаты проведенного нами исследования согласуются с предыдущими работами. Учитывая низкую чувствительность и заниженные значения ИПК, предоперационная оценка вовлеченности брюшины на основании МСКТ не может быть достаточно точной, в то время как комплексная ДВ-МРТ дает результаты, коррелирующие с данными хирургического вмешательства.

В нашем исследовании было установлено, что ДВ-МРТ значительно превосходит МСКТ по способности обнаруживать небольшие метастазы в брюшину и брыжейку. Высокая чувствительность метода связана с сильным ограничением диффрузии в опухолевых очагах из-за высокой клеточной плотности. Однако наличие воздуха и перистальтики ЖКТ может привести к появлению артефактов.

Кроме того, существует ряд эффректов, приводящих к появлению ложноположительных и ложноотрицательных результатов в ДВ-МРТ. В первую очередь, поскольку основой ДВ-МРТ является Т2-взвешенные последовательности, могут наблюдаться следующие эфффекты: Т2 shine-through (просвечивание), Т2 washout (вымывание), T2 blackout (отключение). В первом случае зона имеет гиперинтенсивный сигнал на Т2-взвешенных изображениях, дифффузионновзвешенных изображениях (ДВ-изображения), но не отмечается снижения ИКД (ограничения дифффузии), поэтому одновременное использование ДВ-изображений и карт ИКД позволяет избежать ложноположи- 
тельных результатов. В случае эфффекта Т2-вымывания высокая интенсивность сигнала на Т2-изображениях сочетаться с низкой интенсивностью ДВ-изображения и высокими значениями ИКД, что позволяет исключить ограничение дифффузии. В случае эфффекта Т2-отключения все три характеристики снижены, поэтому об ограничении дифрфузии в очаге можно судить по снижению ИКД, несмотря на низкую интенсивность ДВ-изображений и избежать ложноотрицательных результатов [17]. Важно учитывать, что некоторые ткани (в т.ч., эндометрий, яичники) в норме имеют сниженную дифффузию.

Снижение значений ИКД во вторичных опухолевых очагах может быть связано со снижением диффреренцировки клеток и изменением их фенотипа [24]. В результате в них повышается ядерно-цитоплазматиче- ское соотношение и увеличивается клеточная плотность. Эти фракторы ведут к большему ограничению дифффузии по сравнению с первичным очагом. Кроме того, возможно влияние таких фракторов, как отек и некроз окружающих тканей. Генотипические и фенотипические изменения во вторичных опухолевых очагах могут обуславливать развитие химиорезистентности и высокий риск рецидива опухоли.

\section{Выводы}

ДВ-МРТ значительно превосходит МСКТ по чувствительности и позволяет дать точную оценку распространенности опухолевого процесса, что позволяет эфффективно использовать ДВ-МР-исследование для предоперационного стадирования и оценки резектабельности опухоли у пациенток с РЯ.

\section{Литература:}

1. Siegel R.L., Miller K.D., Jemal A. Cancer statistics, 2015. CA Cancer J Clin. 2015; 65 (1): 5-29

2. Jayson G.C., Kohn E.C., Kitchener H.C., Ledermann J.A. Ovarian cancer. Lancet. 2014; 384: $1376-88$

3. Kurman R.J., Shih I.M. Molecular pathogenesis and extraovarian origin of epithelial ovarian cancer-shifting the paradigm. Hum Pathol. 2011; 42 (7): 918-31

4. Eddie S.L., Quartuccio S.M, Ó hainmhir E., et al. Tumorigenesis and peritoneal colonization from fallopian tube epithelium Oncotarget. 2015; 6 (24): 20500-12.

5. Солопова А.Е., Чащин А.А., Солопова А.Г., Макацария А.Д. Современные взгляды на патогенез и возможности диагностики эпителиального рака яичников.

Акушерство, гинекология и репродукция. 2016; 10 (1): 75-83.

6. Kandukuri S.R., Rao J. FIGO 2013 staging system for ovarian cancer: what is new in comparison to the 1988 staging system. Curr Opin Obstet Gynecol. 2015; 27: 48-52.

7. Mann J.W., Chalas E., Valea F.A. Cancer of the ovary, fallopian tube, and peritoneum: staging and initial management. URL: www.UpToDate.com (2015). Дата обращения: 19.01.2016.

8. Ledermann J.A., Raja F.A., Fotopoulou C., et al. Newly diagnosed and relapsed epithelial ovarian carcinoma: ESMO Clinical Practice Guidelines for diagnosis, treatment and follow-up. Ann Oncol. 2013; 24: 24-32.

9. Chang S.J., Hodeib M., Chang J., Bristow R.E. Survival impact of complete cytoreduction to no gross residual disease for advanced-stage ovarian cancer: a meta-analysis. Gynecol Oncol. 2013; 130 (3): 493-8.

10. Suidan R.S., Ramirez P.T., Sarasohn D.M., et al. A multicenter prospective trial evaluating

\section{References:}

1. Siegel R.L., Miller K.D., Jemal A. Cancer statistics, 2015. CA Cancer J Clin. 2015; 65 (1): 5-29. the ability of preoperative computed tomography scan and serum CA-125 to predict suboptimal cytoreduction at primary debulking surgery for advanced ovarian, fallopian tube, and peritoneal cancer. Gynecol Oncol. 2014; 134 (3): 455-61

11. Kyriazi S., Kaye S.B., Desouza N.M. Imaging ovarian cancer and peritoneal metastasescurrent and emerging techniques. Nat Rev Clin Oncol. 2010; 7 (7): 381-93.

12. Coakley F.V., Choi P.H., Gougoutas C.A., et al. Peritoneal metastases: detection with spiral CT in patients with ovarian cancer. Radiology. 2002; 223 (2): 495-9.

13. Tempany C.M., Zou K.H., Silverman S.G., Brown D.L., Kurtz A.B., Mcneil B.J. Staging of advanced ovarian cancer: comparison of imaging modalities-report from the Radiological Diagnostic Oncology Group. Radiology. 2000; 215 (3): 761-7.

14. Espada M., Garcia-Flores J.R., Jimenez M., et al. Diffusion-weighted magnetic resonance imaging evaluation of intra-abdominal sites of implants to predict likelihood of suboptimal cytoreductive surgery in patients with ovarian carcinoma. Eur Radiol. 2013; 23: 2636-2642.

15. Mukuda N., Fujii S., Inoue C., et al. Apparent diffusion coefficient (ADC) measurement in ovarian tumor: Effect of region-of-interest methods on ADC values and diagnostic ability. J Magn Reson Imaging. 2016; 43 (3): 720-5.

16. Солопова А.Е., Терновой С.К.,

Макацария А.Д., Гуров С.Н. Возможности дисрфузионно-взвешенных последовательностей магнитнорезонансного исследования в оценке эффективности неоадьювантной химиотерапии больных раком яичников. REJR. 2016; 6 (4): 68-80.

17. Gelezhe P.B., Trofimenko I.A., Morozov S.P. The basics of whole-body diffusion-weighted imaging interpretation. REJR. 2015; 5 (3): 65-73.

2. Jayson G.C., Kohn E.C., Kitchener H.C., Ledermann J.A. Ovarian cancer. Lancet 2014; 384: 1376-88.

3. Kurman R.J., Shih I.M. Molecular pathogenesis and extraovarian origin of epithelial ovarian
18. Sugarbaker P.H. Comprehensive management of peritoneal surface malignancy using cytoreductive surgery and perioperative intraperitoneal chemotherapy: the Washington Cancer Institute approach. Expert Opin Pharmacother. 2009; 10 (12): 1965-77.

19. Coffey J.C., O'Leary D.P., The mesentery: structure, function, and role in disease. Lancet Gastroenterol Hepatol. 2016; 1: 238-47

20. Mizumoto A., Canbay E., Hirano M., et al. Morbidity and mortality outcomes of cytoreductive surgery and hyperthermic intraperitoneal chemotherapy at a single institution in Japan. Gastroenterol Res Pract. 2012; 2012: 836425.

21. Casado-adam A., Alderman R., Stuart O.A., Chang D., Sugarbaker P.H. Gastrointestinal complications in 147 consecutive patients with peritoneal surface malignancy treated by cytoreductive surgery and perioperative intraperitoneal chemotherapy. Int J Surg Oncol. 2011; 2011: 468698.

22. Low R.N., Barone R.M., Lucero J. Comparison of MRI and CT for predicting the Peritoneal Cancer Index ( $\mathrm{PCI}$ ) preoperatively in patients being considered for cytoreductive surgical procedures. Ann Surg Oncol. 2015; 22 (5): 1708-15.

23. Michielsen K., Vergote I., Op de beeck K., et al. Whole-body MRI with diffusionweighted sequence for staging of patients with suspected ovarian cancer: a clinical feasibility study in comparison to CT and FDG-PET/CT. Eur Radiol. 2014; 24 (4): 889-901.

24. Oh J.W., Rha S.E., Oh S.N., Park M.Y., Byun J.Y., Lee A. Diffusion-weighted MRI of epithelial ovarian cancers: correlation of apparent diffusion coefficient values with histologic grade and surgical stage. Eur J Radiol. 2015; 84 (4): 590-5.

cancer-shifting the paradigm. Hum Pathol. 2011; 42 (7): 918-31.

4. Eddie S.L., Quartuccio S.M, Ó hainmhir E., et al. Tumorigenesis and peritoneal colonization from fallopian 
tube epithelium. Oncotarget. 2015; 6 (24): 20500-12.

5. Solopova A.E., Chashchin A.A., Solopova A.G., Makatsariya A.D. Akusherstvo, ginekologiya i reproduktsiya / Obstetrics, gynecology and reproduction. 2016; 10 (1): 75-83.

6. Kandukuri S.R., Rao J. FIGO 2013 staging system for ovarian cancer: what is new in comparison to the 1988 staging system. Curr Opin Obstet Gynecol. 2015; 27: 48-52.

7. Mann J.W., Chalas E., Valea F.A. Cancer of the ovary, fallopian tube, and peritoneum: staging and initial management. URL: www.UpToDate.com (2015). Accessed: 19.01.2016.

8. Ledermann J.A., Raja F.A., Fotopoulou C., et al. Newly diagnosed and relapsed epithelial ovarian carcinoma: ESMO Clinical Practice Guidelines for diagnosis, treatment and follow-up. Ann Oncol. 2013; 24: 24-32.

9. Chang S.J., Hodeib M., Chang J., Bristow R.E. Survival impact of complete cytoreduction to no gross residual disease for advancedstage ovarian cancer: a meta-analysis. Gynecol Oncol. 2013; 130 (3): 493-8.

10. Suidan R.S., Ramirez P.T., Sarasohn D.M., et al. A multicenter prospective trial evaluating the ability of preoperative computed tomography scan and serum CA-125 to predict suboptimal cytoreduction at primary debulking surgery for advanced ovarian, fallopian tube, and peritoneal cancer. Gynecol Oncol. 2014; 134 (3): 455-61.

11. Kyriazi S., Kaye S.B., Desouza N.M. Imaging ovarian cancer and peritoneal metastases- current and emerging techniques Nat Rev Clin Oncol. 2010; 7 (7): 381-93.

12. Coakley F.V., Choi P.H., Gougoutas C.A., et al. Peritoneal metastases: detection with spiral CT in patients with ovarian cancer. Radiology. 2002; 223 (2): 495-9.

13. Tempany C.M., Zou K.H., Silverman S.G., Brown D.L., Kurtz A.B., Mcneil B.J. Staging of advanced ovarian cancer: comparison of imaging modalities-report from the Radiological Diagnostic Oncology Group. Radiology. 2000; 215 (3): 761-7.

14. Espada M., Garcia-Flores J.R., Jimenez M., et al. Diffusion-weighted magnetic resonance imaging evaluation of intra-abdominal sites of implants to predict likelihood of suboptimal cytoreductive surgery in patients with ovarian carcinoma. Eur Radiol. 2013; 23: 2636-2642.

15. Mukuda N., Fujii S., Inoue C., et al. Apparent diffusion coefficient (ADC) measurement in ovarian tumor: Effect of region-of-interest methods on ADC values and diagnostic ability J Magn Reson Imaging. 2016; 43 (3): 720-5.

16. Solopova A.E., Ternovoi S.K., Makatsariya A.D., Gurov S.N. REJR. 2016; 6 (4): 68-80.

17. Gelezhe P.B., Trofimenko I.A., Morozov S.P. The basics of whole-body diffusion-weighted imaging interpretation. REJR. 2015; 5 (3): 65-73.

18. Sugarbaker P.H. Comprehensive management of peritoneal surface malignancy using cytoreductive surgery and perioperative intraperitoneal chemotherapy: the Washington Cancer Institute approach. Expert Opin Pharmacother. 2009; 10 (12): 1965-77.
19. Coffey J.C., O'Leary D.P., The mesentery: structure, function, and role in disease. Lancet Gastroenterol Hepatol. 2016; 1: 238-47

20. Mizumoto A., Canbay E., Hirano M., et al. Morbidity and mortality outcomes of cytoreductive surgery and hyperthermic intraperitoneal chemotherapy at a single institution in Japan. Gastroenterol Res Pract. 2012; 2012: 836425.

21. Casado-adam A., Alderman R., Stuart O.A., Chang D., Sugarbaker P.H. Gastrointestinal complications in 147 consecutive patients with peritoneal surface malignancy treated by cytoreductive surgery and perioperative intraperitoneal chemotherapy. Int J Surg Oncol. 2011; 2011: 468698.

22. Low R.N., Barone R.M., Lucero J. Comparison of $\mathrm{MRI}$ and $\mathrm{CT}$ for predicting the Peritoneal Cancer Index (PCl) preoperatively in patients being considered for cytoreductive surgical procedures. Ann Surg Oncol. 2015; 22 (5): 1708-15.

23. Michielsen K., Vergote I., Op de beeck K. et al. Whole-body MRI with diffusionweighted sequence for staging of patients with suspected ovarian cancer: a clinical feasibility study in comparison to CT and FDG-PET/CT. Eur Radiol. 2014; 24 (4): 889-901.

24. Oh J.W., Rha S.E., Oh S.N., Park M.Y., Byun J.Y., Lee A. Diffusion-weighted MRI of epithelial ovarian cancers: correlation of apparent diffusion coefficient values with histologic grade and surgical stage. Eur J Radiol. 2015; 84 (4): 590-5.

\section{Сведения об авторах:}

Солопова Алина Евгеньевна - к.м.н., доцент кафедры лучевой диагностики и лучевой терапии лечебного факультета Первого МГМУ им. И.М. Сеченова. Адрес: ул. Трубецкая, 8, стр. 2, Москва, Россия, 119991. E-mail: dr.solopova@mail.ru. Просриль в ORCID: http://orcid.org/0000-0003-4768-115X.

Терновой Сергей Константинович - д.м.н., академик РАН, профессор, заведующий кафедрой лучевой диагностики и лучевой терапии Первого МГМУ им. И.М. Сеченова. Адрес: ул. Трубецкая, 8, стр. 2, Москва, Россия, 119048. Тел.: +7(499)2487791. E-mail: prof_ternovoy@list.ru. Профииль в ORCID: http://orcid.org/0000-0003-4374-1063.

Алипов Владимир Иванович - студент Первого МГМУ им. И.М. Сеченова. Адрес: ул. Трубецкая, 8, стр. 2, Москва, Россия, 119991. E-mail: alipvladimir@yandex.ru. Профиль в ORCID: http://orcid.org/0000-0002-4430-5258.

Макацария Александр Давидович - д.М.н., член-корреспондент РАН, профессор, заведующий кафедрой акушерства и гинекологии медико-профилактического факультета Первого МГМУ им. И.М. Сеченова. Адрес: ул. Трубецкая, 8, стр. 2, Москва, Россия, 119048. Тел.: +7(495)7885840. E-mail: gemostasis@mail.ru. Профиль в ORCID: http://orcid.org/0000-0002-1395-567X.

\section{About the authors:}

Solopova Alina Evgen'evna - PhD in Medical sciences, Associate Professor of Chair of Radiology and Radiotherapy, Faculty of Medicine, I.M. Sechenov First MSMU. Address: 8/2 Trubetskay st., 119991 Moscow, Russia. E-mail: dr.solopova@mail.ru. A profile in ORCID: http://orcid.org/0000-0003-4768-115X

Ternovoy Sergey Konstantinovich - Grand PhD in Medical Science, Professor, Fellow of the Russian Academy of Sciences, head of Chair of Radiology and Radiotherapy, Faculty of Medicine, I.M. Sechenov First MSMU. Address: 8/2 Trubetskay st., 119991 Moscow, Russia. Phone number: +7(499)2487791. E-mail: prof_ternovoy@list.ru. A profile in ORCID: http://orcid.org/0000-0003-4374-1063

Alipov Vladimir Ivanovich - student of Faculty of Medicine, I.M. Sechenov First MSMU. Postal address: 2-4 Bolshaya Pirogovskaya st., 119048 Moscow, Russia. E-mail: alipvladimir@yandex.ru. A profile in ORCID: http://orcid.org/0000-0002-4430-5258.

Makatsariya Aleksandr Davidovich - Grand PhD in Medical Science, Professor, corresponding member of the Russian Academy of Sciences, head of Chair of Obstetrics and Gynecology, Faculty of Medicine, I.M. Sechenov First MSMU. Postal address: 8/2 Trubetskay st., 119048 Moscow, Russia. Phone number: +7(495)7885840. E-mail: gemostasis@mail.ru. A profile in ORCID: http://orcid.org/00000002-1395-567X. 